\title{
Periodontal management of the elderly with medical compromise: a case report
}

\author{
Ira A. Sembiring, ${ }^{*}$ Andi M. Adam
}

\section{Abstract}

Objective: The aim of the periodontal treatment is to provide healthy and functional dentition all through a lifetime. The primary objective of this case report is to describe that periodontal disease in the elderly complicated by medical compromise should be treated and the results will be very satisfactory.

Methods: A sixty-eight years old female patient with cardiovascular disease took amlodipine and aspirin medication since two months ago. Patients complained of enlarged gingiva, all of the teeth become mobility. Periodontal surgery was performed after referring back to the internist to prepare patient condition facing periodontal surgery.

Keywords: Amlodipine, Cardiovascular disease, Elderly, Hyperplasia gingiva, Periodontal disease

Cite this Article: Sembiring IA, Adam AM. 2019. Periodontal management of the elderly with medical compromise: a case report. Journal of Dentomaxillofacial Science. 4(1): 57-60. D01: 10.15562/jdmfs.v4i1.748

Department of Periodontic, Faculty of Dentistry, Hasanuddin University, Makassar, Indonesia

*Corresponding to: Ira A. Sembiring, Department of Periodontic, Faculty of Dentistry, Hasanuddin University, Makassar, Indonesia iraasnita@yahoo.com

Received: 10 June 2018

Revised: 15 June 2018

Accepted: 16 July 2018

Available online 1 April 2019

\section{Introduction}

The elderly population in Indonesia is the world's top five based on the results of the 2010 population census. Population of elderly in Indonesia reached 20.24 million people, equivalent to 8.03 percent of the total population of Indonesia in $2014 .{ }^{1} 80 \%$ of older people aged over 65 years are diagnosed with one or more chronic diseases and $20 \%$ are limited in their ability to take care of themselves. Chronic diseases such as arthritis, hypertension and respiratory diseases are the most common disorders reported in this age group.

Periodontal pockets are niches that become suitable habitat for hundreds species of bacteria especially periodontal pathogens, resulting inflamed area, which is the starting point of bacteria and/ or by-products into the systemic circulation. ${ }^{2}$ This inflamation disease may further lead to loss of teeth that impair the function of mastication and quality of physical and social. ${ }^{3}$ Several studies identified that periodontitis is a risk factor for systemic disease. . $^{4-8}$

Elderly are also problematic in the social sphere such as poverty, retirement and dependency. Periodontal infection and inflamation are likely to impair oral function, reduce quality of life and increases a patients risk of developing severe chronic systemic disease. ${ }^{3}$ Since the consequences of periodontal disease are critical, general dentist, periodontist and medical specialist practicioner
Results: After several phase of periodontal treatment, the gingival inflamation demonstrated a good control and periodontal status become more stable.

Conclusion: Although managing periodontal disease in elderly is complicated, the proper control of the periodontal infection and inclusion of other healthcare professionals will improve the prognosis and get a satisfactory result. In fact, studies demonstrate that elderly individuals whose comprehensive management includes dental care develop fewer co-morbid conditions and require less expenditure of healthcare rupiahs. 
and root planning was three years ago. A week ago she was visited general dentist to treat her gingival enlargement, and get administered amoxicillin and cataflam.

Generally the patient looks healthy and alert. Intraorally, there was massive gingival enlargement on the labial/palatal of the upper and lower teeth. Her oral hygiene was very poor with abundant plaque and calculus figure 1. Bleeding on probing was detected almost on all region. Periodontal pockets were 3 to $10 \mathrm{~mm}$. From the panoramic radiograph founded multiple retained roots embedded in the overgrown tissues of the upper and lower arch and a few teeth are missing figure 2 . Her upper left central incisors was deeply carious. Most of lower teeth were mobility miller class I and the upper incisors were mobility miller class III.

The prognosis of element 21 and 22 were hopeless because of the bone loss almost two-third root, there were pathologic migration and severe mobility. The diagnosis was chronic periodontitis with gingival hyperplasia induced by medications (amlodipine).

The treatment plan was made, with initial phase is to extract retained root and compromised tooth, instructions of oral hygiene, full mouth scalling root planning, caries removal, restoration and continued with flap surgery if the overgrowth still exists after initial therapy and prosthetic to replace a missing teeth.

Full mouth scaling and root planning of all teeth was done on the first visit and the patient was given oral hygiene instruction and motivation. Referral letter was sent to the internist that treat her to substitute Amlodipine and to get approved for the further treatment such as extraction or/and periodontal surgery. Because of anxiety, patient did not make an appointment scheduled for extraction of hopeless tooth and retained root. After two months, patient come and there is reduction of gingival overgrowth. At the following visit after reexamination, there was still remaining overgrowth gingiva, so that flap surgical and gingivectomy was performed for the lower gingiva. Gingivectomy was done to diminish the fibrous granulated tissue over the interdental region of all gingiva in lower arch. Continued with flap surgery to gain accessibility of scalling and root planning. The retained root at lower arch was extracted. After one week, the gingiva had good improvement, with a slight recession figure 3 .

Second surgery done after one month for region 13 and extraction of retained root at upper arch. One week post surgery, the gingiva had a good result with reduced of pocket depth into normal. Patient are less cooperative because her visits are often hampered by health and anxiety.
The compromised teeth were yet extracted because patients refused the plan. Patient had motivated to eliminate all etiologies of her periodontal disease. Currently this patient will proceed to further treatment.

\section{Discussion}

Periodontal disease is common in elderly throughout the world. In Indonesia, prevalence of periodontal disease in elderly is $86 \%{ }^{11}$ Periodontal disease as a chronic disease had effects that accumulate with age..$^{12}$ Many patients especially elderly seeking periodontal care have significant medical conditions which may alter their treatment plan and management. ${ }^{8}$ Therefore, a thorough medical history and assessment of the patient is a prerequisite to evaluated the impact of a systemic disorder on periodontal therapy. ${ }^{5,6,13}$

Physician consultation is necessary to get detailed information about the systemic condition. It is essential for clinician to have a current level of knowledge of medical conditions and medications and their effects.

Periodontal therapy in elderly must be adjusted to medical conditions, access to care, affordability and the ability to perform adequate oral hygiene. It is important to recognize the need for frequent updates of medical history and medications and to consider the potential risk of complications when providing care to medically compromised patients. Comprehensive treatment plan include adequate patient history (medical/dental), appropriate diagnostic test and physician referral or consultation while indicated. Periodontal treatment in elderly is 'too little, too late' to affect meaningful change for some chronic medical outcomes.

Few studies have assessed the outcome of nonsurgical therapies in people $\geq 65$ years of age. A recent systematic review has shown that older individual that have nonsurgical debridement in the treatment of periodontitis provides moderate improvement in perceived quality of life. ${ }^{4}$

Gingival hyperplasia is characterized by an accumulation of extracellular matrix within the gingival connective tissue particularly the collagenous components. ${ }^{14-16}$ It has been associated with multiple factors including systemic inflammation, adverse drug effects and cardiovascular disease. Drug-induced gingival overgrowth of an adverse drug reaction of commonly prescribed drugs Calcium Channel Blockers (CCBs). Drug-induced gingival hyperplasia usually occurs within the first 3 months of medication and begins as an enlargement of the interdental papilla. ${ }^{17,18}$

Amlodipine, commonly prescribed to treat hypertension, is a third-generation calcium channel 


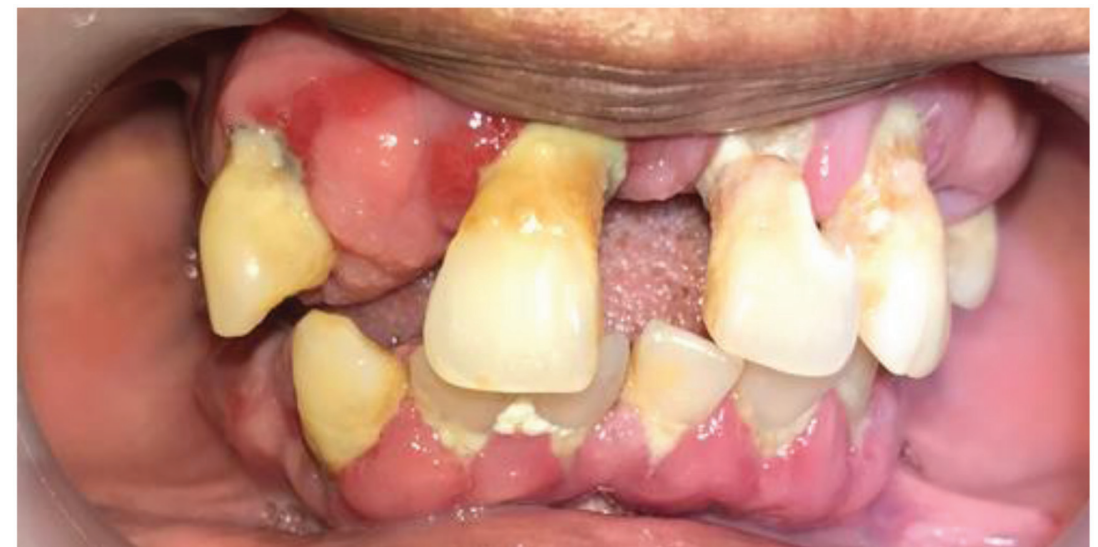

Figure 1 Enlargement gingiva with poor oral hygiene at first visit

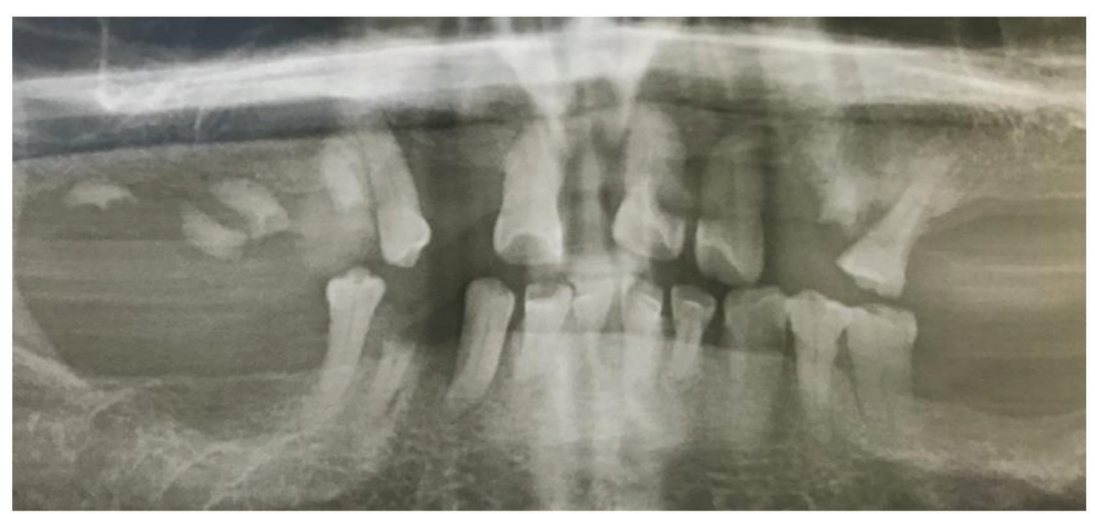

Figure 2 Panoramic radiograph view

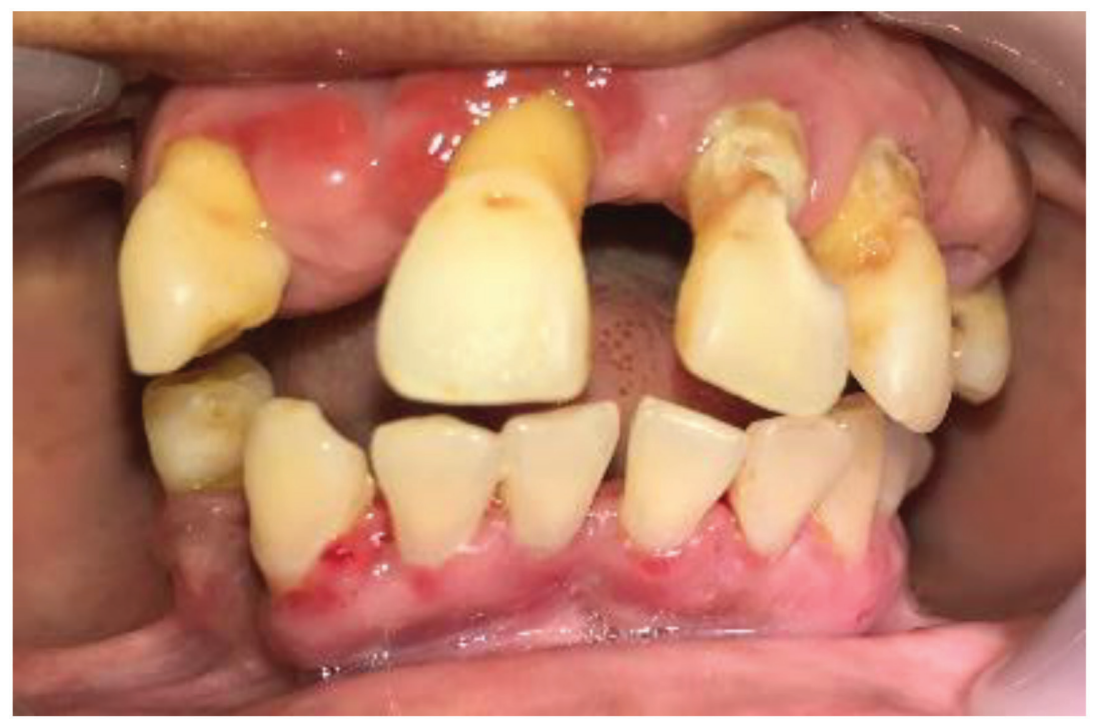

Figure 3 After periodontal surgery

antagonist. Amlodipine advantages over firstgeneration calcium channel antagonists such as nifedipine; because of fewer systemic side effects such as hypotension, peripheral oedema, nausea, palpitation and syncope and it is generally better tolerated. $^{18}$
The exact mechanism behind the effect of amlodipine and the increased risk of gingival hyperplasia is not clearly understood. Amlodipine affect calcium ion cellular flux, that makes plausible for amlodipine increase the risk of gingival hyperplasia. Amlodipine inhibit the intercellular uptake of calcium and this action may change the gingival fibroblasts secretory properties or the production of collagenases. The result is overstimulated gingival fibroblasts. However, consider many studies this condition were significant determined by poor oral hygiene and plaque-induced inflammation. ${ }^{18,19}$

To produce a healthy periodontium that characterized by a lack of bleeding on probing and shallowing pocket $(\leq 3 \mathrm{~mm})$, dentist must be pursued whenever there is a chance to success, it may not be an achievable outcome in many elderly patient. This compromise should not be regarded as being sub-standard in any way. The dentist must have a realistic treatment outcome in mind before commences the periodontal therapy. However the outcome may vary depending upon the patient wish and individual findings.

How effective is periodontal therapy in elderly, it is certainly worth while treating periodontal disease in elderly with high quality treatment combined with adequate plaque control and motivation to result in a good therapeutic outcome. ${ }^{20,21}$ The literature suggest that age per se is not an important factor in determining the result of periodontal therapy. ${ }^{19}$

\section{Conclusion}

Periodontal disease has been linked to so many chronic medical diseases. Despite periodontal treatment of elderly may be too late, too little to affect meaningful change for some chronic medical outcomes, but proven by literature increased optimal oral health that is substantial for reduction of the risk for chronic diseases and increased a quality of life.

Periodontal treatment whether surgical and non surgical therapy for elderly is possible. Management of periodontal disease in elderly with medically compromise is a worthwhile cause the patient in this case report is proven can be executed and the results are good and satisfactory.

\section{Acknowledgment}

The authors would like to thank M. Ruslin Acing Habibie Mude and Fuad Husain Akbar, whom have had supported this article. 


\section{Conflict of Interest}

The authors report no conflict of interest.

\section{References}

1. Badan Pusat Statistika. Statistik penduduk lanjut usia. Stat Pendud Lanjut usia 2014: 1-239.

2. Socransky SS. Periodontal microbial ecology. EBSCO host 2005;38: 135-187.

3. Palomo JM. Periodontal diseases and quality of life. Biomed J Sci Tech Res 2017;1: 10-12.

4. Persson GR. Dental geriatrics and periodontitis. Periodontol 2000 2017;74: 102-115.

5. Lamster IB. Geriatric periodontology: how the need to care for the aging population can influence the future of the dental profession. Periodontol 2000 2016;72: 7-12.

6. Scannapieco FA, Cantos A. Oral inflammation and infection, and chronic medical diseases: implications for the elderly. Periodontol 2000 2016;72: 153-175.

7. Ajwani S. Periodontal disease in an aged population, and its role in cardiovascular mortality. Helsinki: Department of Medicine, Helsinki University Central Hospital; 2003. p. 3.

8. L.R-Malo, Bullon P. Review influence of the periodontal disease, the most prevalent inflammatory event, in peroxisome proliferator-activated receptors linking nutrition and energy metabolism. Int J Molecullar Sci 2017;18: 1438.

9. Boehm TK, Scannapieco FA. The epidemiology, consequences and management of periodontal disease in older adults. JADA 2007;138: S26-S34.

10. Cafiero C. Review article periodontal care as a fundamental step for an active and healthy ageing. Scien World J 2017.

11. Bahruddin T, Dian A. Status jaringan periodontal dan kebutuhan perawatan jaringan periodontal pada manula Suku Bugis dan Suku Mandar. J Dentomaxillofac Sci 2007;6: 51-57.
12. Tonetti MS. Dental caries and periodontal diseases in the ageing population: call to action to protect and enhance oral health and well-being as an essential component of healthy ageing-Consensus report of group 4 of the joint EFP/ORCA workshop on the boundaries between caries and periodontal diseases. J Clin Periodontol 2017;44 (suppl 18): s135-s144.

13. Renvert S, Persson GR. Treatment of periodontal disease in older adults. Periodontol 2000 2016;72: 108-119.

14. Kaur G. Association between calcium channel blockers and gingival hyperplasia. J Clin Periodontol 2010;37: 625-630.

15. Amit B, Shalu BV. Gingival enlargement induced by anticonvulsants, calcium channel blokers, immunouppressants: Review. Int Res J Pharmacy 2012;3: 116-119.

16. Chouksey A, Awasthi N, Rai J, et al. Association of druginduced gingival enlargement (calcium channel blockers) and local factors: Who is the culprit?. Int Dent \& Med J Adv Res 2017;3: 1-4.

17. Bagtzoglou AD. Drug-associated gingival enlaegement. J Periodontol 2004;75: 1424-1431.

18. Chaturvedi R, Jain A. Amlodepine induced gingival enlargement-presentation of a clinical case series. J Clin Exp Dent 2011;3(Suppl1): e390-394.

19. Gilbert AD. Management of periodontal disease in elderly patient. Periodontol 2006;3: 195-203.

20. Narhi T, Syrjala AM. Dental diseases and their treatment in the older population. TandlÆgebladet 2017: 121-151.

21. Chou YH, Yang YH, Kuo HC, et al. Periodontal surgery improves oral health-related quality of life in chronic periodontitis patients in Asian population. Kaohsiung J Med Sci 2017;33: 523-529.

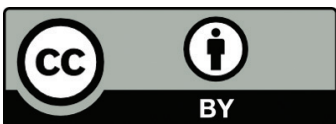

This work is licensed under a Creative Commons Attribution 